\title{
Paeoniflorin suppresses allergic and inflammatory responses by promoting autophagy in rats with urticaria
}

\author{
JING GUO $^{1^{*}}$, LI PENG $^{1 *}$, JINHAO ZENG $^{2,3}$, MEIHENG ZHANG ${ }^{1}$, \\ FENG XU ${ }^{1}$, XIAOTONG ZHANG ${ }^{1}$ and QIN WEI ${ }^{1}$ \\ ${ }^{1}$ Dermatological Department, ${ }^{2}$ Geriatric Department, ${ }^{3}$ TCM Regulating Metabolic Diseases Key Laboratory of \\ Sichuan Province, Hospital of Chengdu University of Traditional Chinese Medicine, Chengdu, Sichuan 610072, P.R. China
}

Received February 6, 2020; Accepted July 1, 2020

DOI: $10.3892 /$ etm.2021.10022

\begin{abstract}
Paeoniflorin (PF) has been reported to be effective against several skin disorders, such as allergic contact dermatitis and psoriasis; however, it remains unclear whether PF can protect against urticarial lesions. Herein, the effects of PF on rats with urticarial lesions and the possible underlying mechanism were investigated. The effects of PF administration on a rat model of ovalbumin-induced urticarial-like lesions were evaluated via pathological analysis using hematoxylin-eosin staining. Toluidine blue staining was performed to detect mast cells and ELISA was performed to determine serum histamine levels. PF-induced regulatory effects on autophagic activity and the potential underlying mechanism of this were also investigated using transmission electron microscopy, immunohistochemistry and reverse transcription-quantitative PCR. It was demonstrated that PF suppressed allergic and inflammatory responses to improve urticarial lesions, as evidenced by the attenuation of pathological abnormalities, mast cell infiltration and histamine secretion. Mechanistically, PF treatment was found to markedly limit the production and release of inflammatory cytokine interleukin (IL)-23, while the levels of IL-17 remained unchanged. PF intervention led to an increased number of autophagosomes, along with higher levels of light chain 3B (LC3B) and Beclin-1, and lower levels of P62, indicating that PF could augment autophagic activity in urticarial lesions. PF treatment increased the expression of liver kinase B1 (LKB1) and AMP-activated protein kinase- $\alpha$ (AMPK- $\alpha$ ), contributing to the PF-enhanced autophagic activity. In conclusion, PF could effectively improve urticarial
\end{abstract}

Correspondence to: Dr Jinhao Zeng, TCM Regulating Metabolic Diseases Key Laboratory of Sichuan Province, Hospital of Chengdu University of Traditional Chinese Medicine, 39 Shi-er-qiao Road, Chengdu, Sichuan 610072, P.R. China

E-mail: zengjinhao@cdutcm.edu.cn

*Contributed equally

Key words: urticaria, paeoniflorin, autophagy, inflammation, allergy lesions by inhibiting inflammatory cytokine IL-23 and increasing the autophagic activity via the LKB1/AMPK- $\alpha$ pathway.

\section{Introduction}

Urticaria, also known as hives, is a common condition estimated to affect up to $20 \%$ of the general population during their lifetime (1). Frequent episodes of urticaria can impact patients' lives directly, through itching, and indirectly, by interfering with their sleep and daily activities. While $\mathrm{H} 1$ antihistamines were used as first-line treatment, certain reported adverse events and poorly responsive cases have been associated with long-term use of the drugs, particularly in patients with chronic urticarial (2), necessitating the development of novel and efficient alternative therapies.

Paeoniflorin (PF) is a monoterpene glucoside extracted from the root of Paeonia lactiflora Pall. In China, 'total glucosides of paeony' capsule, of which $\mathrm{PF}$ is the major component, was approved for the treatment of rheumatoid arthritis by the National Medical Products Administration (approval no. H20055058), and was recently recommended as a novel treatment for psoriasis by the China Association of Chinese Medicine (3). Previously, PF had been shown to be a safe extraction used in clinical pharmacological research (4). Experimentally, PF offered protection against UV-induced DNA damage in human keratinocytes (5), and could ameliorate several skin disorders, such as allergic contact dermatitis (6) and psoriasis $(7,8)$. In addition, studies have also revealed that PF possess anti-inflammatory (6), antioxidative (9), antiapoptotic (10) and immunoregulatory bioactivities (11). It was proposed herein that PF may serve as a promising candidate for urticaria treatment.

Autophagy is a fundamental cellular pathway through which cytoplasmic organelles and intracellular pathogens undergo degradation. The autophagic process is closely associated with the immune-inflammatory response (12): Autophagy regulates both innate and adaptive immunity (13). Furthermore, cytokines produced during the course of the immune response modulate various functions of the autophagic cascade (14). An essential role of autophagy is its involvement in the inflammatory pathways in allergic disease, which was supported by recent data showing that greater airway inflammation and 
autophagy activation, marked by a higher Beclin-1 and ATG-5 expression and lowed P62 expression, was identified in human asthmatic tissues (15). Moreover, the induction of autophagy in $\mathrm{CX} 3 \mathrm{Crl}^{+}$mononuclear phagocytes inhibited the expression of interleukin (IL)-23, leading to a reduced fibrotic response (16). Conversely, the inhibition of autophagy augmented IL-23 secretion in dendritic cells and supernatants from these cells stimulated the innate secretion of IL-17 (17). A recent study showed that a moisturizer with autophagy-stimulating properties contributed to skin barrier restoration and control of inflammation, thereby alleviating the symptoms and signs of atopic dermatitis in patients (18). To date, however, few studies have directly linked autophagy to urticarial lesions.

In the present study, an ovalbumin (OVA)-induced urticarial rat model was used to examine whether PF could effectively protect against urticarial lesions. Subsequently, the current study sought to determine whether and how PF affected the levels of IL-23/IL-17 in the rats with urticaria. The possibility of PF-induced regulatory effects on autophagic activity and the potential underlying mechanism were also investigated.

\section{Materials and methods}

Animals. A total of 40 male Sprague-Dawley rats (8 weeks old) were obtained from Chengdu Dashuo Experimental Animal Co., Ltd. (license no. SCXK-2015-030). Animals were housed in a specific pathogen-free animal room, kept under optimal conditions of $22 \pm 2^{\circ} \mathrm{C}$ and $40-60 \%$ humidity under a 12-h light/dark cycle with ad libitum access to food and water. Animal health and behavior were monitored on a daily basis by the research and animal care staff. The animal experiment was performed at the Animal Experimental Center, West China Hospital, Sichuan University (Chengdu, China), all animal welfare considerations were taken, and all procedures were approved by the Institutional Animal Care and Use Committee of West China Hospital of Sichuan University (approval no. 2019236A).

Drugs. PF (purity, $\geq 98 \%$ ) was purchased from Beijing Solarbio Science and Technology Co., Ltd. (cat. no. IP0030). Loratadine (LOR) was supplied from Bayer Pharmaceutical (Shanghai) Co., Ltd. (H10970410).

Animal model and treatments. After 1 week of acclimation, the animals were randomly assigned to four groups as follows ( $=10 /$ group): i) Normal group treated with saline; ii) model group treated with OVA/alum (ChenDu Chron Chemicals Co., Ltd.; cat. no. 21645-51-2) and saline; iii) model + LOR group treated with OVA/alum and $0.9 \mathrm{mg} / \mathrm{kg}$ LOR; and iv) model + PF group treated with OVA/alum and $40 \mathrm{mg} / \mathrm{kg}$ PF. To develop urticarial lesions, the animals, except the normal controls, were sensitized intraperitoneally (i.p.) with $1 \mathrm{ml}$ aluminum hydroxide suspension (alum dissolved in $10 \mathrm{~g} / 10.9 \% \mathrm{NaCl}$ ) containing $1 \mathrm{mg}$ OVA (cat. no. S7951; Sigma-Aldrich; Merck KGaA) on days 0, 2 and 4, and challenged i.p. with $2 \mathrm{mg}$ OVA in alum suspension on day 14. Following the third sensitization, the normal and model rats were treated with vehicle, while model $+\mathrm{LOR}$ and model $+\mathrm{PF}$ rats were administered orally with LOR and PF, respectively, on days 5-14, once daily. All animals were humanely euthanatized with sodium pentobarbital (140 mg/kg i.p.) $3 \mathrm{~h}$ after the final challenge, which was performed $1 \mathrm{~h}$ after oral administration. Then, blood samples were collected by orbital puncture, shaved dorsal skin tissues were harvested.

Pathological analysis. Shaved dorsal skin was fixed in $4 \%$ paraformaldehyde overnight at $4^{\circ} \mathrm{C}$, embedded in paraffin and cut into $5-\mu \mathrm{m}$-thick sections. The sections were stained at room temperature with hematoxylin for $5 \mathrm{~min}$ and eosin (H\&E) for 2 min to investigate pathological changes. Three fields of view per slide were observed under a light microscope (IX71; Olympus Corporation) and images were captured. The magnifications used were $\mathrm{x} 100$ and $\mathrm{x} 400$. The sections were evaluated by two independent pathologists in a blinded manner.

Mast cell detection. Toluidine blue (TB) staining was performed for mast cell detection (number and degranulation), which were clearly recognizable due to the stained red-purple granules. Shaved dorsal skin was fixed in $4 \%$ paraformaldehyde overnight at $4^{\circ} \mathrm{C}$, embedded in paraffin and cut into $5-\mu \mathrm{m}$-thick sections. The sections were dipped and stained with $0.5 \%$ toluidine blue solution for $30 \mathrm{~min}$ at room temperature. Subsequently, the sections were washed with distilled water and separated for $5 \mathrm{sec}$ in $0.5 \%$ glacial acetic acid solution, after which samples were dehydrated in a gradient alcohol series, transparent xylene and neutral gum. Three random visual fields per slide were selected and photographed under a light microscope (IX71; Olympus Corporation). Mast cells in each field were counted, and average number of the three field cells was presented as the mast cell number per rat. The magnification used was x100.

Serum histamine levels. The blood samples were centrifuged at $3,500 \mathrm{x} \mathrm{g}$ for $10 \mathrm{~min}$ at $4^{\circ} \mathrm{C}$ and then serum was collected and stored at $-80^{\circ} \mathrm{C}$ for quantitative analysis. According to the manufacturer's instructions, the serum concentrations of histamine were detected using a rat histamine ELISA kit (cat. no. K4163; BioVision, Inc.). Absorbance was measured at a wavelength of $450 \mathrm{~nm}$.

Transmission electron microscopy (TEM). Dorsal skin tissues were fixed with $2.5 \%$ glutaraldehyde in cacodylate buffer (pH 7.4) overnight at $4^{\circ} \mathrm{C}$, and then post-fixed in $2 \%$ osmium tetroxide in the same buffer. Next, the specimens were conventionally dehydrated in acetone, infiltrated and embedded in epoxy resin-filled capsules. Finally, 70-nm ultrathin sections were prepared with a ultramicrotome (LKB Instruments) and counterstained with $2 \%$ aqueous uranyl acetate and $0.8 \%$ lead citrate at $25^{\circ} \mathrm{C}$ for $3 \mathrm{~h}$. Ultrastructures were examined and imaged using a transmission electron microscope (H-7650; Hitachi, Ltd.). The number of autophagic vacuoles per unit field at a magnification of x12,000 was counted, and three randomly selected fields of view were captured for each sample.

Immunohistochemistry (IHC). Skin tissues were fixed in $4 \%$ paraformaldehyde for $24 \mathrm{~h}$ at room temperature, embedded in paraffin and cut into $5 \mu \mathrm{m}$-thick sections. Sections were then deparaffinized in xylene for $10 \mathrm{~min}$, hydrated through a series of graded alcohol $(30,50,70,95$ and $100 \%)$, and microwaved in citrate buffer ( $\mathrm{pH} 6.0$ ) for $10 \mathrm{~min}$ at $95^{\circ} \mathrm{C}$. Following routine 
peroxidase blocking with $3 \%$ hydrogen peroxide solution for 15 min and 5\% bovine serum albumin blocking (cat. no. G5001; Wuhan Servicebio Technology Co., Ltd.) for $30 \mathrm{~min}$ at room temperature, the sections were incubated with antibodies against IL-23 (dilution, 1:200; cat. no. orb184437; Biorbyt Ltd.), IL-17 (dilution, 1:200; cat. no. NBP1-42746; Novus Biologicals, LLC), Beclin-1 (dilution, 1:100; cat. no. PD017; MBL International Co.), light chain 3B (LC3B; dilution, 1:200; cat. no. NB100-2220; Novus Biologicals, LLC), P62 (dilution, 1:100; cat. no. ab56416; Abcam), liver kinase B1 (LKB1; dilution, 1:150; cat. no. CPA3329; Cohesion Biosciences, Ltd.) and AMP-activated protein kinase- $\alpha$ (AMPK- $\alpha$; dilution, 1:100; cat. no. 66536-1-1g; ProteinTech Group, Inc.) overnight at $4^{\circ} \mathrm{C}$. The samples were then exposed to HRP/Fab polymer-conjugated secondary antibodies (solution from the kit; cat. no. PV-6000-D; OriGene Technologies, Inc.) at room temperature for $30 \mathrm{~min}$. Samples were then stained with 3,3'-diaminobenzidine solution for $5 \mathrm{~min}$ and counterstained with hematoxylin for $20 \mathrm{sec}$ at room temperature.

Each slide was analysed using light microscopy and three random fields of view were observed. Images were captured and analyzed using Image Pro Plus 6.0 software (Media Cybernetics, Inc.). The mean of integrated optical density was used to evaluate the semi-quantitative levels of IL-23, IL-17, Beclin-1, LC3B, P62, LKB1 and AMPK- $\alpha$. The magnification used was $\mathrm{X} 100$.

Reverse transcription-quantitative PCR (RT-qPCR) analysis. RNA from skin tissues was extracted using the TRIzol ${ }^{\circledR}$ reagent (cat. no. 15596026; Thermo Fisher Scientific, Inc.), and cDNA was synthesized using the RevertAid First Strand cDNA Synthesis kit (cat. no. K1621; Thermo Fisher Scientific, Inc.) according to the manufacturer's protocol. Real Time PCR Easy ${ }^{\mathrm{TM}}$-SYBR Green I (cat. no. QP-01014; Foregene Co., Ltd.) was used as fluorophore. Next, RT-qPCR of the samples was determined using the real-time PCR detection system (PikoReal 96-well Thermal Cycler; Thermo Fisher Scientific, Inc.). The following thermocycling conditions were used: Initial denaturation for $30 \mathrm{sec}$ at $95^{\circ} \mathrm{C} ; 45$ cycles of $5 \mathrm{sec}$ at $95^{\circ} \mathrm{C}, 30 \mathrm{sec}$ at $55^{\circ} \mathrm{C}$ and $30 \mathrm{sec}$ at $72^{\circ} \mathrm{C}$. The mRNA levels of IL-23, IL-17, Beclin-1, LC3B, P62, LKB1 and AMPK- $\alpha$ were analyzed. $\beta$-actin was used as an endogenous control to normalize the amounts of RNA between samples. Differences in amplification were calculated using the $2^{-\Delta \Delta \mathrm{Cq}}$ method (19). The primer sequences used are listed in Table I.

Statistical analysis. Statistical analysis was performed using SPSS 17.0 software (SPSS, Inc.). Differences between groups were evaluated using one-way analysis of variance, followed by Tukey method for data with equal variances and Dunnett's T3 method for data with unequal variances. Data are expressed as the mean $\pm \mathrm{SD} . \mathrm{P}<0.05$ was considered to indicate a statistically significant difference.

\section{Results}

PF ameliorates urticarial histopathology. H\&E staining was first conducted to evaluate the pathological features in dorsal skin from various groups. Pathological abnormalities were clearly visible in urticarial lesion biopsies in model rats,
Table I. Primer sequences

\begin{tabular}{ll}
\hline Primer name & \multicolumn{1}{c}{ Sequence (5'-3') } \\
\hline$\beta$-actin & F: GAAGATCAAGATCATTGCTCCT \\
& R: TACTCCTGCTTGCTGATCCA \\
IL-23 & F: ACCTGCTGGACTCGGACATCTTCACA \\
& R: AAGGCTTGGAGGCTGCGAAGGATCT \\
IL-17 & F: TGTGCCTGATGCTGTTGCTGCTACTG \\
& R: GGTCCTCATTGCGGCTCAGAGTCCA \\
Beclin-1 & F: GTCTAAGGCGTCCAGCAGCACCAT \\
& R: GGTCACTCGGTCCAGGATCTTGAAGC \\
LC3B & F: GCTGCCTGTCCTGGATAAGACCAAGT \\
& R: CCTCCTCTTGACTCAGAAGCCGAAGG \\
P62 & F: GCTGAGTCGGCTTCTGCTCCATCA \\
& R: GCGGCTTCTCTTCCCTCCATGTTCC \\
LKB1 & F: AGAGGAGGAGGAGGACGAGGACTTGT \\
& R: CCTTCTGGCTTCACCTTGCTGCTGAG \\
AMPK- $\alpha$ & F: CGATGATGAGGTGGTGGAGCAGAGGT \\
& R: GTGAATGGTTCTCGGCTGTGCTGGAA
\end{tabular}

$\mathrm{F}$, forward; R, reverse; IL, interleukin; LC3B, light chain 3B; LKB1, liver kinase B1; AMPK- $\alpha$, AMP-activated protein kinase- $\alpha$.

compared with skin in healthy controls. Dorsal skin from model rats exhibited apparent edema in the upper and mid dermis, forming separated collagenous fibers that had faded in color, as well as widened spaces between collagen bundles. Prominent dilated post-capillary venules surrounded with infiltration of some inflammatory cells were also observed. PF treatments could notably attenuate these pathological abnormalities observed in model rats (Fig. 1). Marked pathological mitigation was also observed in LOR-treated rats compared with the model group. These findings indicated that PF intervention allowed for a remission of urticarial histopathology.

PF inhibits mast cell infiltration and degranulation. Mast cell infiltration and degranulation are the central events in type I allergic responses (20), which are responsible for active urticaria. TB staining was therefore performed to examine whether PF could be effective in ameliorating mast cell activation. In the present study, the number of mast cells and granules released (Fig. 2A) was significantly higher in model $(53.10 \pm 4.45)$ than in normal rats $(8.57 \pm 1.16)$, suggesting that an apparent type I allergic response had occurred in the rats with urticaria. By contrast, the number of mast cells and granules decreased significantly in rats following LOR or PF treatment (Fig. 2). The administration of PF was able to markedly attenuate the infiltration of mast cells and suppress the elevated degranulation responses.

PF decreases serum histamine levels. Histamine is a key mediator in the inflammatory response, largely due to mast cell activation $(21,22)$. Therefore, serum histamine levels were determined herein using ELISA. Histamine levels in rats with urticaria were found to be evidently elevated $(38.45 \pm 1.15)$ compared with the control rats $(12.77 \pm 0.86)$, along with 

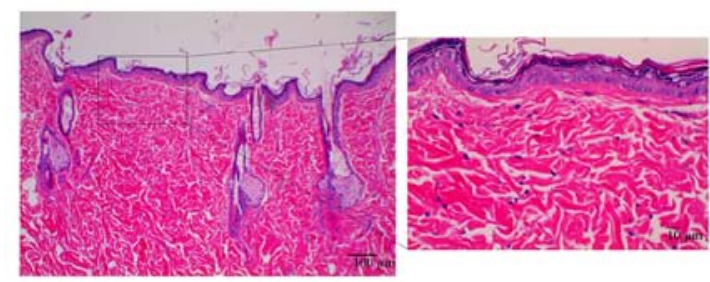

Normal

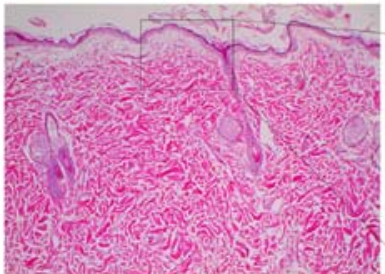

Model+LOR
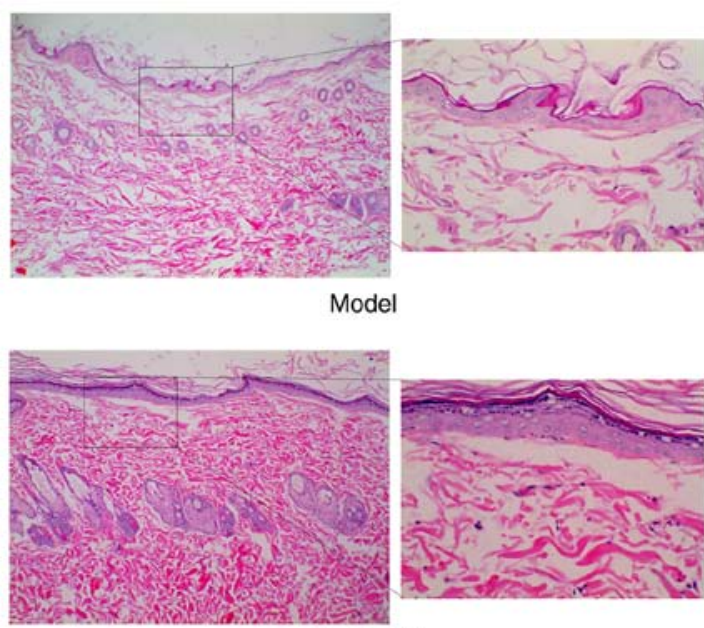

Model+PF

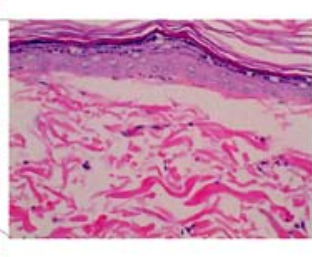

model urticarial and LOR and PF-treated Figure $1 . \mathrm{PF}$ ameliorates histopathological abnormalities in rats with urticaria. Dorsal skin obtained from normal, model urticariation,
rats with urticaria were stained using hematoxylin and eosin (magnification, x100 and x400). PF, paeoniflorin; LOR, loratadine.

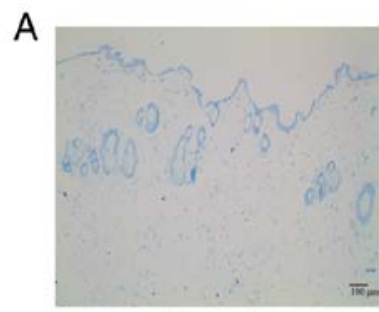

Normal

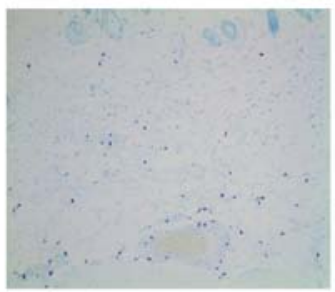

Model

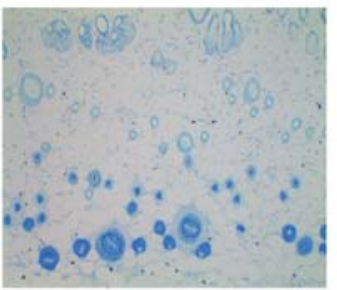

Model+LOR

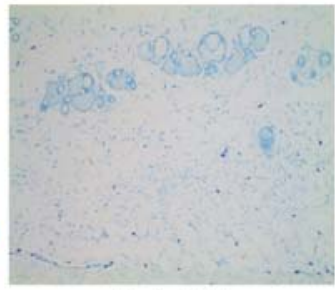

Model+PF

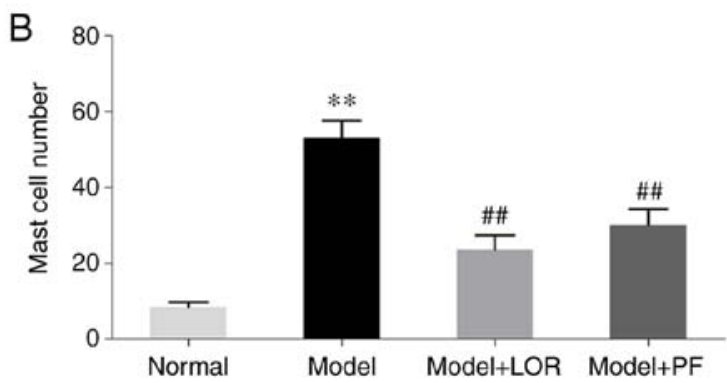

Figure 2. PF inhibits mast cell infiltration and degranulation in rats with urticaria. (A) Representative images of toluidine blue-stained mast cells in dorsal skin tissue (magnification, x100). (B) Number of mast cells in the dermis $(\mathrm{n}=10) .{ }^{* *} \mathrm{P}<0.01$ vs. normal group; ${ }^{\# \#} \mathrm{P}<0.01$ vs. model group. Data are presented as the mean \pm SD. PF, paeoniflorin; LOR, loratadine.

the aforementioned elevation in mast cell number and degranulation. By contrast, PF treatment, which was akin to the effectiveness of LOR, significantly lowered the increased histamine levels (Fig. 3). The application of PF was able to inhibit the overproduction of mast-cell-released histamine.

$P F$ diminishes $I L-23$ production in rats with urticaria. Several studies have revealed the key role that IL-23/IL-17 play in promoting the development of skin lesions infiltrated with inflammatory cells in several diseases, such as psoriasis (23), atopic dermatitis (24) and allergic contact dermatitis (25). Whether IL-23- and IL-17-producing cells could also be observed in urticarial lesions was investigated in the current study. Detection of IL-23 and IL-17 protein by IHC confirmed a significant increase in the number of IL-23-positive cells in dorsal skin sections from rats with urticaria, while there were

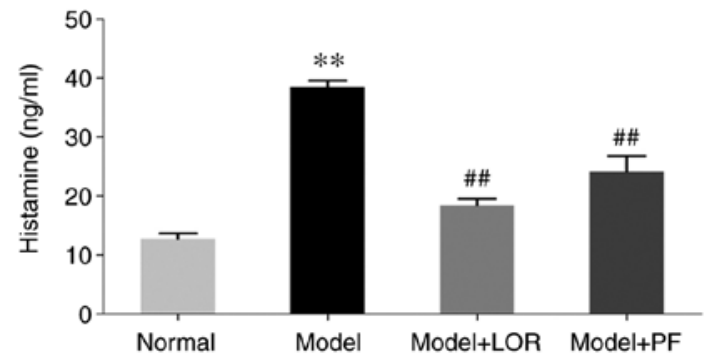

Figure 3. PF decreases serum histamine level in rats with urticaria. ${ }^{* *} \mathrm{P}<0.01$ vs. normal group; ${ }^{\# \#} \mathrm{P}<0.01$ vs. model group. Data are presented as the mean $\pm \mathrm{SD}(\mathrm{n}=10)$. PF, paeoniflorin; LOR, loratadine.

no noticeable changes in the number of IL-17-positive cells (data not shown). PF treatment markedly reduced the number of IL-23-positive cells and PF outperformed LOR in diminishing 


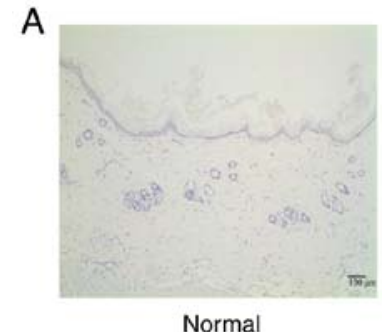

Normal

B

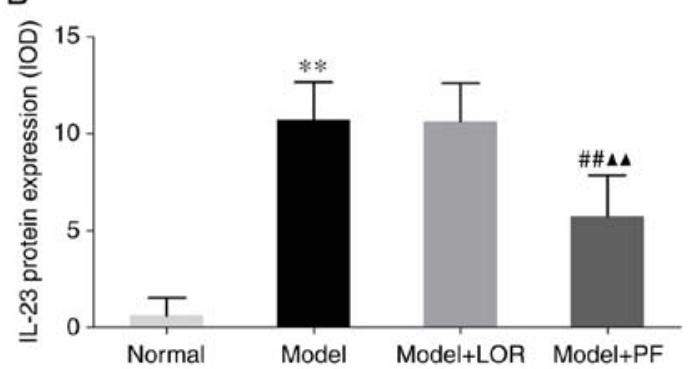

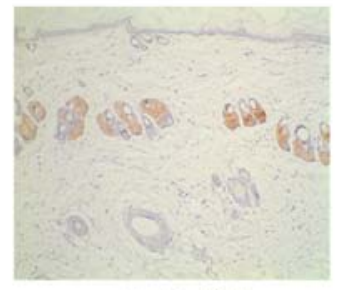

Model+LOR

C

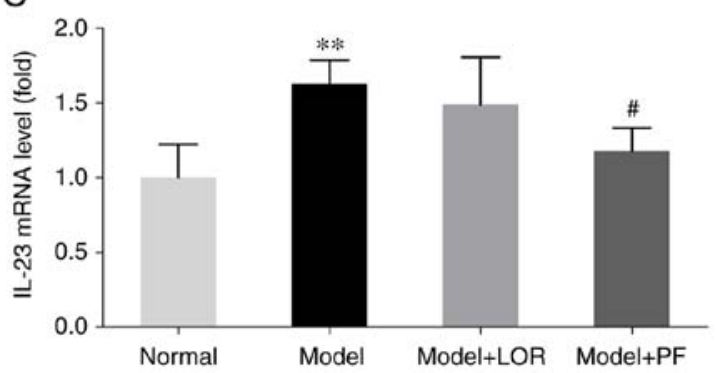

Figure 4. Effects of PF on IL-23 production in rats with urticaria. (A) Representative IHC images showing IL-23 expression in dorsal skin sections from each group (magnification, x100). (B) Semi-quantitative analysis of IL-23 protein expression using IHC (n=10). (C) Reverse transcription-quantitative PCR of IL-23 mRNA levels in each group $(\mathrm{n}=6)$. ${ }^{* *} \mathrm{P}<0.01$ vs. normal group; ${ }^{\#} \mathrm{P}<0.05$ and ${ }^{\# \#} \mathrm{P}<0.01$ vs. model group; ${ }^{\boldsymbol{\Lambda}} \mathrm{P}<0.01$ vs. model $+\mathrm{LOR}$ group. Data are presented as the mean \pm SD. PF, paeoniflorin; IL, interleukin; IHC, immunohistochemistry; LOR, loratadine; IOD, integrated optical density.

the production of IL-23 (Fig. 4A and B). Subsequently, RT-qPCR analysis was performed, and it was found that rats with urticaria displayed a higher amount of IL-23 mRNA than normal rats, which was also significantly lower following PF or LOR treatment (Fig. 4C). However, PF demonstrated lower levels of mRNA and protein IL-23 expression compared with LOR treatment, further supporting the effectiveness of PF in attenuating the inflammatory response partly conferred by IL-23 inhibition.

$P F$ enhances autophagic activity in rats with urticaria. To test whether a disturbed process of autophagy was associated with urticarial activity, and whether PF had a regulatory effect on the autophagic activity, autophagic morphology was first examined in dorsal skin using TEM. As presented in Fig. 5A, the nucleus, mitochondria, endoplasmic reticulum and other organelles appeared morphologically intact in normal rats. In model rats with urticaria, by contrast, cell shrinkage, chromatin condensation and nuclear fragmentation were observed. The presence of swollen mitochondria with broken cristae, or mitochondrial vacuolization and expanded endoplasmic reticulum were also noted. In addition, an increased number of autophagic vacuoles engulfing structure-clear organelles or degraded electron-dense material were found, indicating the presence of elevated autophagic activity in model rats with urticaria. Of note, the autophagic activity was further enhanced by the co-administration of antigenic stimulation with PF, ascertained by the observation that PF-treated rats had $\sim 2$-fold more autophagosomes than non-treated model rats (Fig. 5B). Of note, aberrant morphological ultrastructures, including nuclear fragmentation, chromatin condensation, vacuolated cytoplasm and degenerated cell organelles, were simultaneously improved in the majority of rats treated with PF. However, there was no statistical difference between the LOR treatment group and the model group. Accordingly, PF intervention could, in effect, promote autophagic activity and also help restore cellular ultrastructure in rats with urticaria.

$P F$ increases LC3B and Beclin-1 levels and reduces P62 levels in rats with urticaria. Autophagic activity at the molecular level was further examined by detecting the expression of autophagy markers LC3B, Beclin-1 and P62 in dorsal skin tissues. Beclin-1 is an essential autophagy effector, which forms a complex with PI3K, accounting for autophagic vesicle nucleation in mammals (26). Antigen induction promoted an increase in Beclin-1 levels in rats with urticaria. In addition, treatment with PF had an additive effect in enhancing Beclin-1 levels, as confirmed by RT-qPCR and IHC (Fig. 6A-C). PF may function to promote the production of Beclin-1, contributing to an enhancement in autophagic vacuole formation.

LC3B sensitively reflects autophagic activity and is considered as a specific reporter of autophagic flux (27). Therefore, LC3B mRNA and protein levels were assayed in skin tissues. Consistent with the varying numbers of autophagic vacuoles visualized under TEM, RT-qPCR results suggested that antigen induction led to a significant increase in LC3B content, which could be further, markedly boosted by PF treatment (Fig. 6E). This result was also confirmed by similar trends in LC3B protein expression evidenced by IHC (Fig. 6A and D). Moreover, LOR treatment led to increased LC3B protein expression in rats with urticaria (Fig. 6A and D).

The impact of PF on $\mathrm{P} 62$, an adaptor protein playing a crucial role in the proteasome or lysosome degradation system, was subsequently explored. In the present study, following antigenic stimulation, rats with urticaria demonstrated a reduction of P62 mRNA and protein levels in the dorsal skin, and rats with urticaria treated with $\mathrm{PF}$ achieved a greater decrease in the amount of P62 (Fig. 6A, F and G). Collectively, these findings further supported the TEM observation that PF may have a beneficial effect, given the pathological remission yielded by 


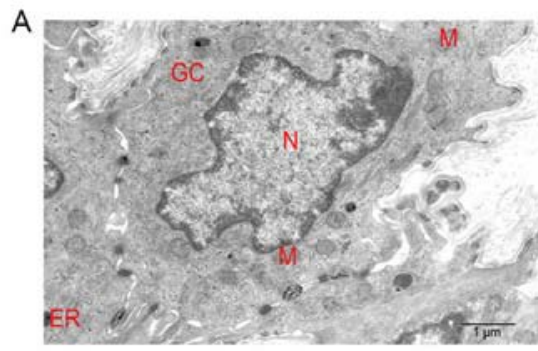

Norma

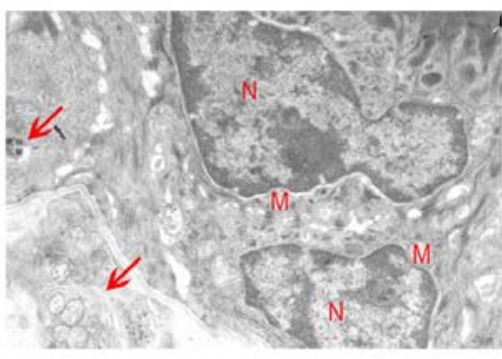

Model+LOR

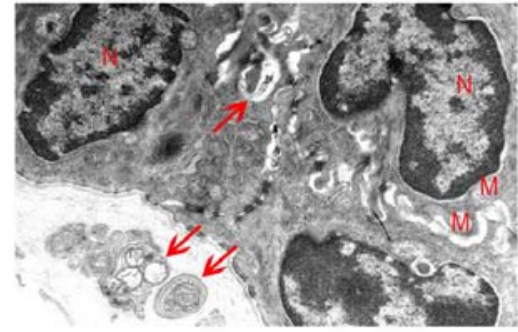

Model

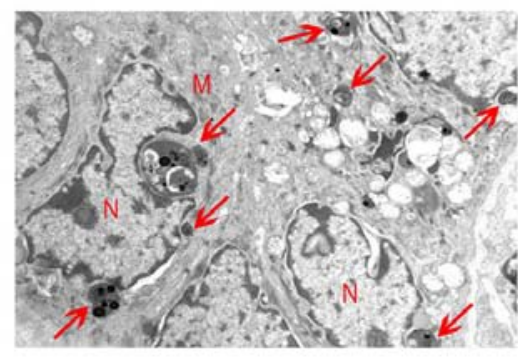

Model+PF

B

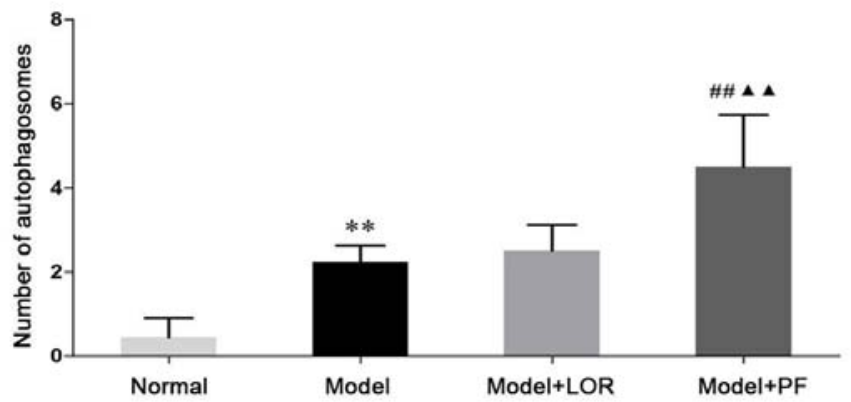

Figure 5. PF enhances autophagic activity in rats with urticaria. (A) Representative transmission electron microscopy images illustrating autophagosomes (red

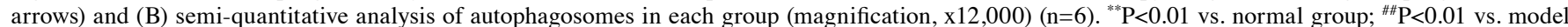
group; ${ }^{\wedge} \mathrm{P}<0.01$ vs. model + LOR group. Data are presented as the mean $\pm \mathrm{SD}$. PF, paeoniflorin; LOR, loratadine; N, nucleus; M, mitochondrion; ER, endoplasmic reticulum; GC, golgi complex.

PF intervention, through promoting autophagic activity in rats with urticaria.

PF activates the LKB1/AMPK signaling pathway in rats with urticaria. LKB1/AMPK is a canonical upstream signaling pathway that modulates autophagic activity $(28,29)$. Whether LKB1/AMPK was involved in PF-enhanced autophagy in rats with urticaria was therefore investigated. The RT-qPCR results indicated that antigenic stimulation led to an increase in the LKB1 and AMPK- $\alpha$ mRNA expression (although no statistical significance was showed for LKB1, $\mathrm{P}=0.053$ ), which could be further, significantly boosted by PF treatment (Fig. 7C and E). IHC also showed an increased protein expression of LKB1 and AMPK- $\alpha$ in dorsal skin sections of rats with urticaria, compared with normal rats, and PF treatment further boosted the LKB1 and AMPK- $\alpha$ expression (Fig. 7A, B and D). Accordingly, the activation of LKB1/AMPK- $\alpha$ may be implicated in the role of PF-enhanced autophagic activity in mitigating urticarial lesions.

\section{Discussion}

Urticaria is generally considered to be a mast cell-driven disease. During a urticaria episode, activated skin mast cells release histamine and other mediators, including leukotriene, platelet-activating factor and cytokines (1), thereby leading to the dilatation and augmentation of the permeability of vessels, plasma extravasation, as well as inflammatory cell recruitment to the urticarial lesions. In the present study, histopathological examination of the affected skin showed apparent edema, dilated capillary and post-capillary venules, as well as a perivascular infiltration of inflammatory cells, including neutrophils, eosinophils and, in some cases, macrophages. All of the above were evidence of salient features of urticaria occurrence. An increased number of mast cells in the skin and elevated concentrations of mast-cell-released histamine were also observed. PF, similar to the effectiveness of LOR, could notably improve urticarial histopathology and ameliorate the overproduction of histamine. Considering that a positive association was noted between the histamine-releasing activity and severity of histopathological features, it may be hypothesized that PF possess anti-allergic properties partly by alleviating mast cell infiltration and degranulation and partly by normalizing histamine release.

IL-23 is a heterodimeric cytokine that plays a pivotal role in the inflammatory autoimmune pathogenesis of multiple skin diseases. IL-23 is one of the essential mediators for driving and maintaining the differentiation of $\mathrm{T}$ helper 

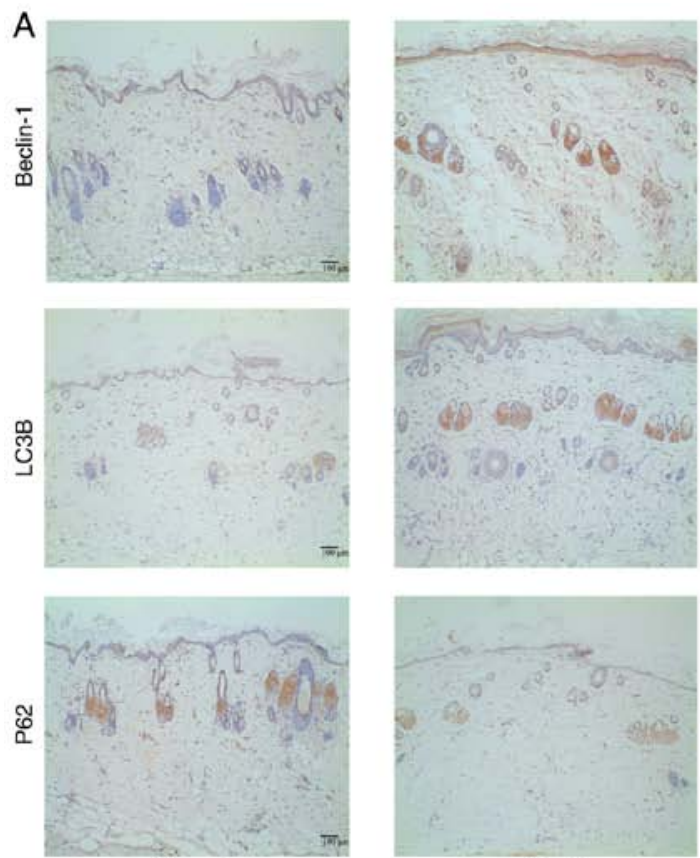

Normal

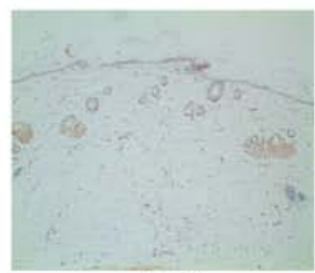

Model

B

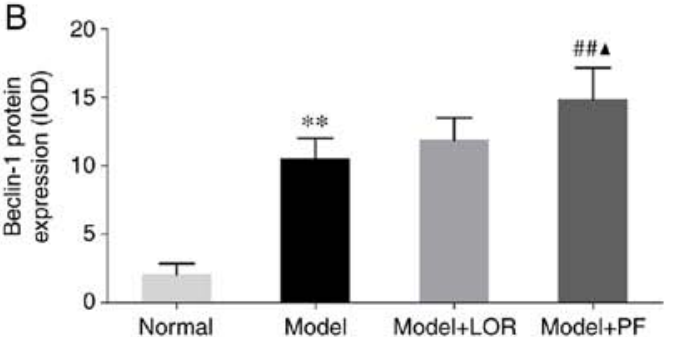

D

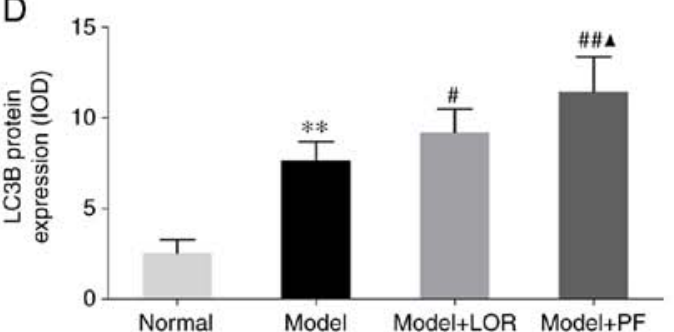

G

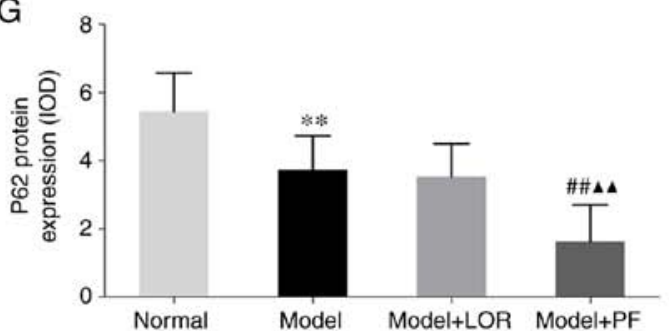

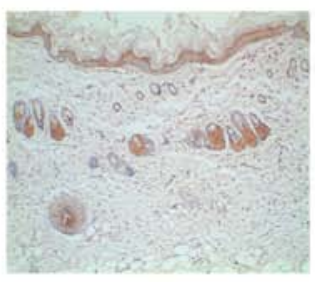
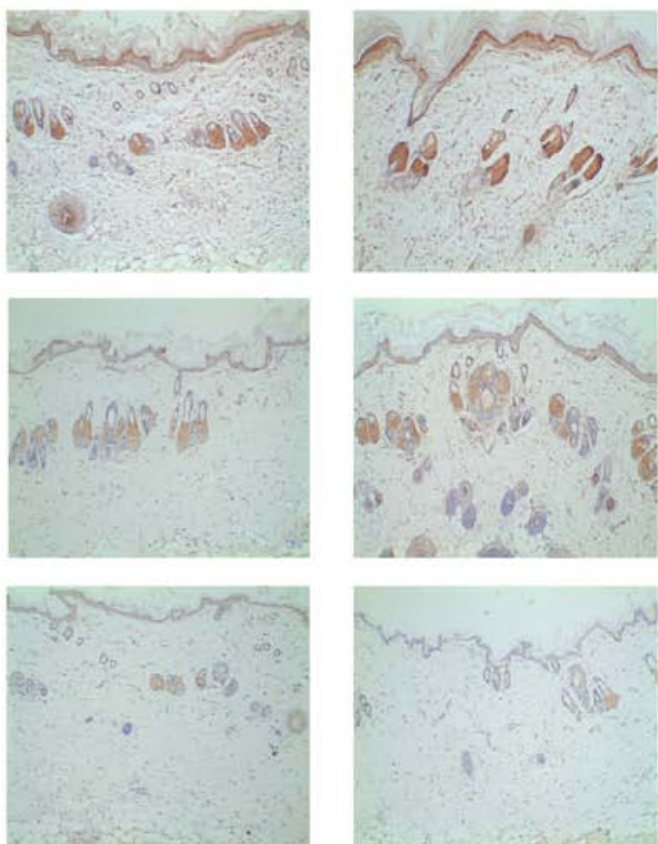

Model+LOR

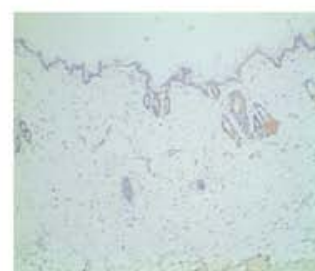

Model+PF

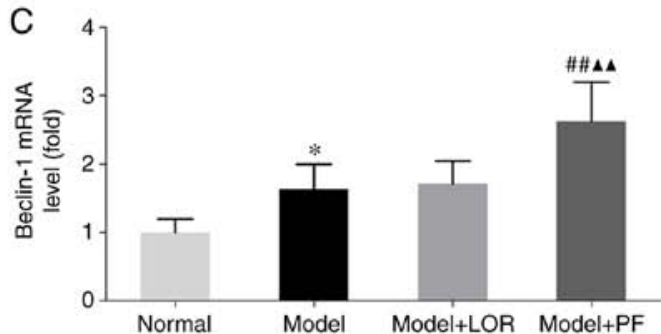

E

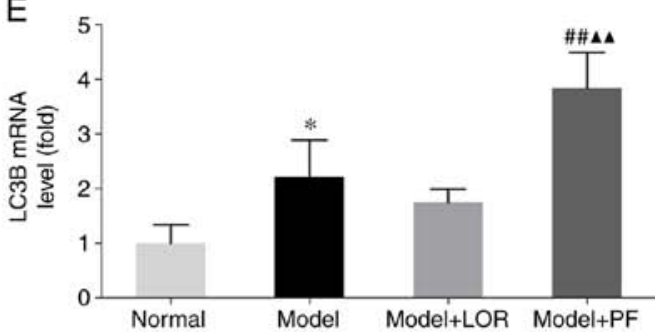

$\mathrm{F}$

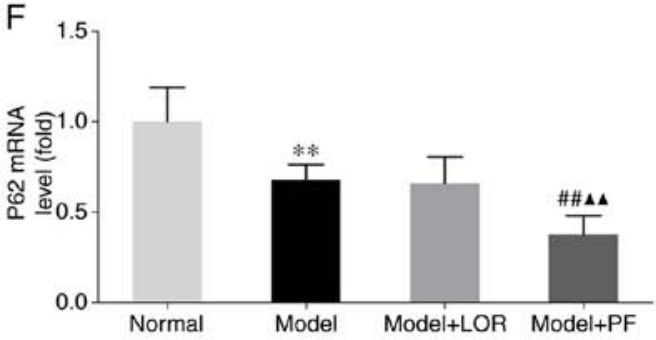

Figure 6. Effects of PF on Beclin-1, LC3B and P62 in rats with urticaria. (A) Representative IHC images showing the expression of Beclin-1, LC3B and P62 in dorsal skin sections from each group (magnification, x100). Semi-quantitative analysis of (B) Beclin-1, (D) LC3B and (F) P62 protein expression using IHC $(n=10)$. Reverse transcription-quantitative PCR of $(C)$ Beclin-1, $(E)$ LC3B and $(G) P 62 m R N A$ levels in each group ( $\mathrm{m}=6)$. * $<0.05$ and ${ }^{* * *} \mathrm{P}<0.01$ vs. normal group; ${ }^{\#} \mathrm{P}<0.05$ and ${ }^{\# \#} \mathrm{P}<0.01$ vs. model group; ${ }^{\boldsymbol{\Delta}} \mathrm{P}<0.05$ and ${ }^{\boldsymbol{\Delta}} \mathrm{P}<0.01$ vs. model $+\mathrm{LOR}$ group. Data are presented as the mean $\pm \mathrm{SD}$. $\mathrm{PF}$, paeoniflorin; LC3B, light chain 3B; IHC, immunohistochemistry; LOR, loratadine.

17 lymphocytes $(30,31)$, which is the resource for producing another effector cytokine IL-17. Using a genetic mouse model, researchers revealed that keratinocyte-produced IL-23 was sufficient to cause chronic skin inflammation (32). Treatment with anti-IL-23p19 and anti-IL-17A neutralizing antibodies improved diabetic wounds harboring excessive inflammation (33). In addition, evidence has emerged that specific inhibition or blockage of IL-23 or IL-17 could improve inflammatory skin disorders, such as psoriasis (34), palmoplantar pustulosis (35) and pityriasis rubra pilaris (36). In the 

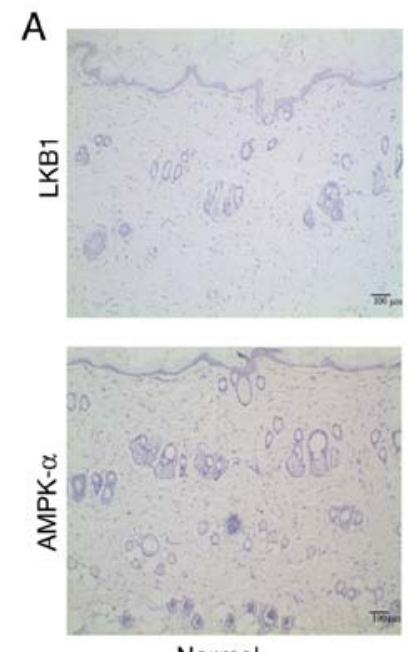

Normal

B

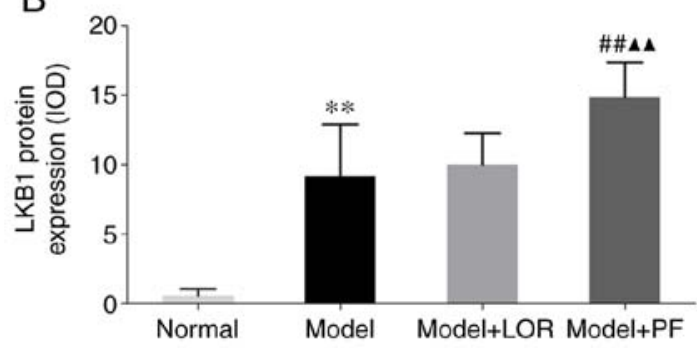

D

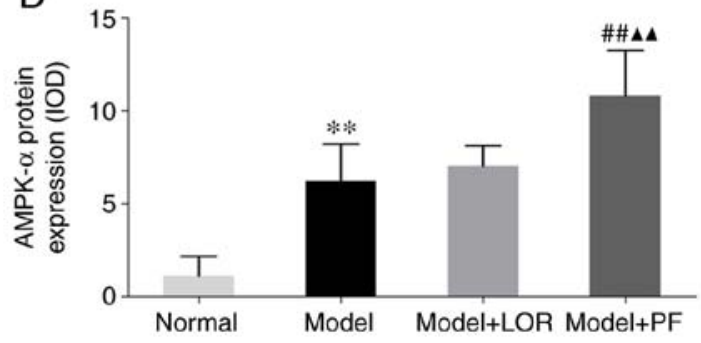

Model
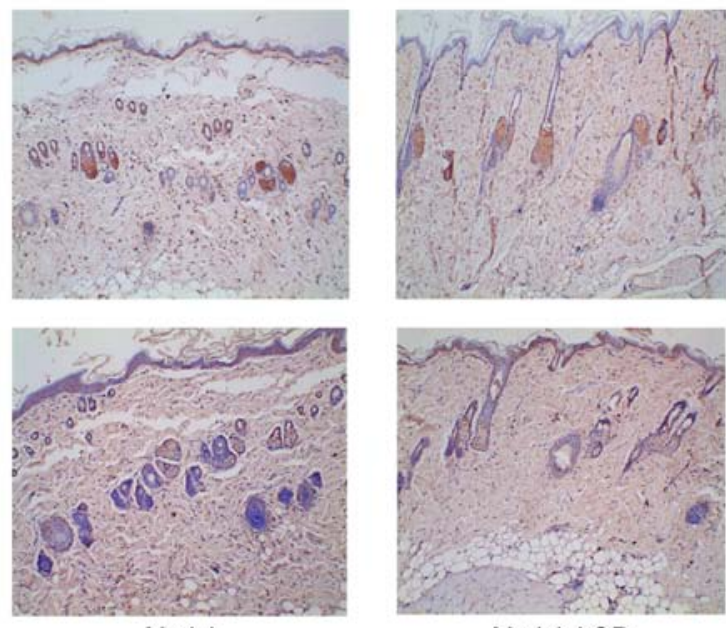

Model+LOR
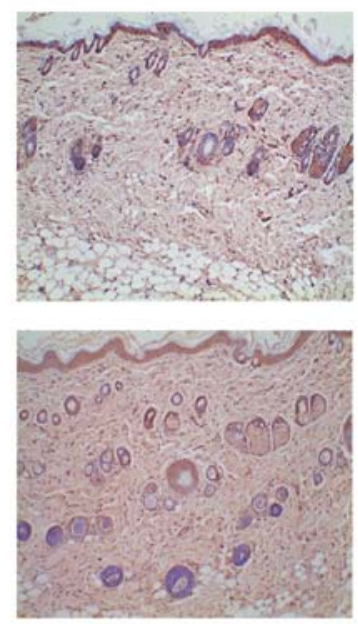

Model+PF

C

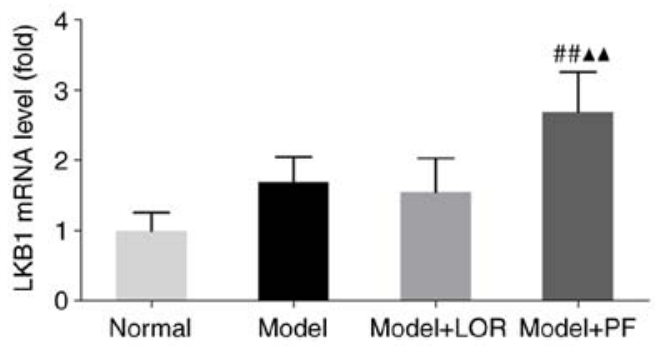

E

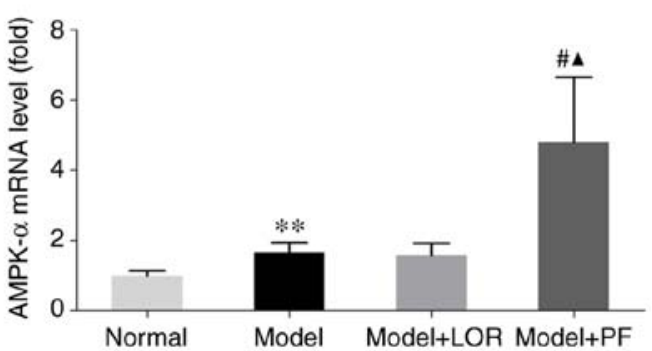

Figure 7. Effects of PF on LKB1 and AMPK- $\alpha$ in rats with urticaria. (A) Representative IHC images showing the expression of LKB1 and AMPK- $\alpha$ in dorsal skin sections from each group (magnification, x100). Semi-quantitative analysis of (B) LKB1 and (D) AMPK- $\alpha$ protein expression using IHC (n=10). Reverse transcription-quantitative PCR of (C) LKB1 and (E) AMPK- $\alpha$ mRNA levels in each group (n=6). ${ }^{* *} \mathrm{P}<0.01$ vs. normal group; ${ }^{\#} \mathrm{P}<0.05$ and ${ }^{\# \# ~} \mathrm{P}<0.01$ vs. model group; ${ }^{\boldsymbol{\Delta}} \mathrm{P}<0.05$ and ${ }^{\boldsymbol{\Delta}}{ }^{\boldsymbol{}} \mathrm{P}<0.01$ vs. model + LOR group. Data are presented as the mean $\pm \mathrm{SD}$. PF, paeoniflorin; LKB1, liver kinase B1; AMPK- $\alpha$, AMP-activated protein kinase- $\alpha$; IHC, immunohistochemistry; LOR, loratadine.

present study, the elevated protein and mRNA expression of IL-23 was noted in OVA-challenged rats with urticaria, which might be a major mechanism underlying inflammatory cell recruitment and infiltration in urticarial lesions. This increased secretion of IL-23 was diminished significantly following PF intervention, thus contributing to the remission of urticarial lesions.

The protective mechanisms of PF against OVA-elicited urticaria were also investigated in our study. Autophagy is crucial to the degradation (37) and recycling of cellular components, and occurs physiologically at a low basal level, aiming at performing homeostatic functions. In the present study, along with the intact nucleus, mitochondria, endoplasmic reticulum and other organelles, autophagosomes were occasionally present in normal skin cells, as determined by TEM. This observation supports the notion that a normal biogenesis and function of autophagic vacuoles is essential for protein and organelle turnover in physiological states (38). P62 binds directly to autophagosomal membrane protein LC3B and presents P62-containing protein aggregates to the autophagy machinery, facilitating the clearance of such aggregates and, thereby, contributing to the process of autophagy $(39,40)$. Lysosomal degradation of autophagosomes has been shown to give rise to reduced P62 levels during autophagy. Conversely, autophagy inhibition has been shown to lead to an increase in P62 protein levels (39). Of note, rats with urticaria exhibited an increased number of autophagosomes, also revealed by the unregulated levels of LC3B, suggesting that skin cells may undergo an increased autophagic process, and hence remove the impairing cytoplasmic components caused by allergic reactions. In the current study, administration of PF further promoted the autophagic activity in model rats as demonstrated by a higher number of autophagosomes together with higher LC3B and lower P62 levels. It was also found that 
the IL-23 reduction occurred in parallel with the degree of pathological remission, as well as the elevation of autophagic activity in PF-treated rats. In previous studies, LKB1 signaling was found to trigger and modulate autophagy by activating AMPK $(28,29,41)$. Pharmacological intervention with PF has been reported to relieve certain diseases by activating the LKB1/AMPK signaling pathway. A recent study found that PF protected against intestinal ischemia/reperfusion by activating LKB1/AMPK and thereby promoting autophagic activity (42). In addition, the beneficial effects of PF in the treatment of insulin resistance and hepatic steatosis were associated with the activation of LKB1/AMPK- $\alpha$ (43). In the present study, PF treatment could boost the expression of LKB1 and AMPK- $\alpha$, contributing to the enhancement of autophagy. Collectively, the results of the current study indicated that PF may confer an augmented autophagic activity, which, in part, could be linked to the activation of LKB1/AMPK- $\alpha$ in urticarial lesions, so as to abrogate IL-23-associated skin inflammatory responses.

Overall, the present study demonstrated the benefits of PF in the treatment of OVA-induced urticaria. However, there were also certain limitations. For instance, only a single dose of PF was administered during urticaria treatment and a detailed understanding of the pharmacodynamics of PF, such as dose-dependent effects, is further required. In addition, further studies that focus on characterizing the association between the autophagic activity and inflammatory disorders after PF treatment are required.

In conclusion, the present study provided evidence that PF serves as an effective antagonist normalizing the IL-23 secretion and ameliorating urticaria, and revealed the novel finding that PF can increase autophagic activity that involves LKB1/ AMPK- $\alpha$ activation, thereby possibly accelerating the elimination of the elicited inflammatory mediators. The present study extended the understanding of the pharmaceutical functions of PF and may provide experimental evidence for its potential clinical use in urticaria treatment.

\section{Acknowledgements}

Not applicable.

\section{Funding}

This work was funded by the National Natural Science Foundation of China (grant nos. 81573986 and 81873310), the Project of Science and Technology Department of Sichuan Province (grant no. 2018JY0660), the Project of 'Xing-lin Scholars' of Chengdu University of Traditional Chinese Medicine (grant nos. CGZH2018001 and QNXZ2019017), Science and Technology Developmental Foundation of the Hospital of Chengdu University of Traditional Chinese Medicine (grant no. 19TS03) and 'Hundred Talents Program' of the Hospital of Chengdu University of Traditional Chinese Medicine (grant nos. 20-B01, 20-Q03 and 20-Q05).

\section{Availability of data and materials}

The datasets used and/or analyzed during the current study are available from the corresponding author on reasonable request.

\section{Authors' contributions}

JG conceived and designed the research, and interpreted the results of the experiments. LP and JHZ designed the current study. LP, FX and MHZ performed the experiments. XTZ and QW conducted the statistical analysis. JHZ and LP organized the database prepared the manuscript. JG and JHZ confirm the authenticity of all the raw data. All authors read and approved the final manuscript.

\section{Ethics approval and consent to participate}

This study was approved by the Institutional Animal Care and Use Committee of West China Hospital, Sichuan University, Chengdu, China (approval no. 2019236A).

\section{Patient consent for publication}

Not applicable.

\section{Competing interests}

The authors declare that they have no competing interests.

\section{References}

1. Saini SS: Chronic spontaneous urticaria: Etiology and pathogenesis. Immunol Allergy Clin North Am 34: 33-52, 2014.

2. Maurer M, Staubach P, Raap U, Richter-Huhn G, Bauer A, Ruëff F, Jakob T, Yazdi AS, Mahler V, Wagner N, et al: H1-antihistamine-refractory chronic spontaneous urticaria: It's worse than we thought - first results of the multicenter real-life AWARE study. Clin Exp Allergy 47: 684-692, 2017.

3. Zheng Q, Jiang W, Sun X, Ma T, Xu W, Shen F, Li H, Xie S, Li B and Li X: Total glucosides of paeony for the treatment of psoriasis: A systematic review and meta-analysis of randomized controlled trials. Phytomedicine 62: 152940, 2019.

4. Li X, Shi F, Zhang R, Sun C, Gong C, Jian L and Ding L: Pharmacokinetics, safety, and tolerability of amygdalin and paeoniflorin after single and multiple intravenous infusions of huoxue-tongluo lyophilized powder for injection in healthy chinese volunteers. Clin Ther 38: 327-337, 2016.

5. Lee S, Lim JM, Jin MH, Park HK, Lee EJ, Kang S, Kim YS and Cho WG: Partially purified paeoniflorin exerts protective effects on UV-induced DNA damage and reduces facial wrinkles in human skin. J Cosmet Sci 57: 57-64, 2006.

6. Wang C, Yuan J, Wu HX, Chang Y, Wang QT, Wu YJ, Liu LH and Wei W: Paeoniflorin inhibits inflammatory responses in mice with allergic contact dermatitis by regulating the balance between inflammatory and anti-inflammatory cytokines. Inflamm Res 62: 1035-1044, 2013.

7. Sun Y, Zhang J, Huo R, Zhai T, Li H, Wu P, Zhu X, Zhou Z, Shen $\mathrm{B}$ and $\mathrm{Li} \mathrm{N}$ : Paeoniflorin inhibits skin lesions in imiquimod-induced psoriasis-like mice by downregulating inflammation. Int Immunopharmacol 24: 392-399, 2015.

8. Chen T, Fu LX, Zhang LW, Yin B, Zhou PM, Cao N and Lu YH: Paeoniflorin suppresses inflammatory response in imiquimod-induced psoriasis-like mice and peripheral blood mononuclear cells (PBMCs) from psoriasis patients. Can J Physiol Pharmacol 94: 888-894, 2016.

9. Choi EM, Suh KS, Rhee SY and Kim YS: Inhibitory effect of paeoniflorin on methylglyoxal-mediated oxidative stress in osteoblastic MC3T3-E1 cells. Phytomedicine 21: 1170-1177, 2014.

10. Wang D, Wong HK, Feng YB and Zhang ZJ: Paeoniflorin, a natural neuroprotective agent, modulates multiple anti-apoptotic and pro-apoptotic pathways in differentiated PC12 cells. Cell Mol Neurobiol 33: 521-529, 2013.

11. Zhang T, Yang Z, Yang S, Du J and Wang S: Immunoregulatory effects of paeoniflorin exerts anti-asthmatic effects via modulation of the Th1/Th2 equilibrium. Inflammation 38: 2017-2025, 2015. 
12. Levine B and Kroemer G: Autophagy in the pathogenesis of disease. Cell 132: 27-42, 2008.

13. Levine B, Mizushima N and Virgin HW: Autophagy in immunity and inflammation. Nature 469: 323-335, 2011.

14. Orosz L, Papanicolaou EG, Seprenyi G and Megyeri K: IL-17A and IL-17F induce autophagy in RAW 264.7 macrophages. Biomed Pharmacother 77: 129-134, 2016.

15. Racanelli AC, Kikkers SA, Choi AMK and Cloonan SM: Autophagy and inflammation in chronic respiratory disease. Autophagy 14: 221-232, 2018.

16. Mathur R,Alam MM,Zhao XF,Liao Y, Shen J, Morgan S, Huang T, Lee H, Lee E, Huang Y and Huang Y: Induction of autophagy in $\mathrm{Cx}_{3} \mathrm{cr}^{+}$mononuclear cells limits IL-23/IL-22 axis-mediated intestinal fibrosis. Mucosal Immunol 12: 612-623, 2019.

17. Peral de Castro C, Jones SA, Ni Cheallaigh C, Hearnden CA, Williams L, Winter J, Lavelle EC, Mills KH and Harris J: Autophagy regulates IL-23 secretion and innate T cell responses through effects on IL-1 secretion. J immunol 189: 4144-4153, 2012.

18. Kwon SH, Lim CJ, Jung J, Kim HJ, Park K, Shin JW, Huh CH, Park KC and Na JI: The effect autophagy-enhancing peptide in moisturizer on atopic dermatitis: A randomized controlled trial. J Dermatolog Treat 30: 558-564, 2019.

19. Livak KJ and Schmittgen TD: Analysis of relative gene expression data using real-time quantitative PCR and the 2(-Delta Delta C(T)) method. Methods 25: 402-408, 2001.

20. Ying S, Kikuchi Y, Meng Q, Kay AB and Kaplan AP: TH1/TH2 cytokines and inflammatory cells in skin biopsy specimens from patients with chronic idiopathic urticaria: Comparison with the allergen-induced late-phase cutaneous reaction. J Allergy Clin Immunol 109: 694-700, 2002.

21. Sun B, Wang B and Xu M: Esculetin inhibits histamine-induced expression of inflammatory cytokines and mucin in nasal epithelial cells. Clin Exp Pharmacol Physiol 46: 821-827, 2019.

22. Metz M and Maurer M: Mast cells-key effector cells in immune responses. Trends Immunol 28: 234-241, 2007.

23. Hawkes JE, Yan BY, Chan TC and Krueger JG: Discovery of the IL-23/IL-17 signaling pathway and the treatment of psoriasis J Immunol 201: 1605-1613, 2018.

24. Mizutani N, Sae-Wong C, Kangsanant S, Nabe T and Yoshino S Thymic stromal lymphopoietin-induced interleukin-17A is involved in the development of IgE-mediated atopic dermatitis-like skin lesions in mice. Immunology 146: 568-581, 2015.

25. Larsen JM, Bonefeld CM, Poulsen SS, Geisler C and Skov L: IL-23 and T(H)17-mediated inflammation in human allergic contact dermatitis. J Allergy Clin Immunol 123: 486-492, 2009.

26. Kihara A, Kabeya Y, Ohsumi Y and Yoshimori T: Beclin-phosphatidylinositol 3-kinase complex functions at the trans-Golgi network. EMBO Rep 2: 330-335, 2001.

27. Kabeya Y, Mizushima N, Ueno T, Yamamoto A, Kirisako T, Noda T, Kominami E, Ohsumi Y and Yoshimori T: LC3, a mammalian homologue of yeast Apg8p, is localized in autophagosome membranes after processing. EMBO J 19: 5720-5728, 2000

28. Jin P, Jiang J, Xie N, Zhou L, Huang Z, Zhang L, Qin S, Fu S, Peng L, Gao W, et al: MCT1 relieves osimertinib-induced CRC suppression by promoting autophagy through the LKB1/AMPK signaling. Cell Death Dis 10: 615, 2019.

29. Zhang M, Deng YN, Zhang JY, Liu J, Li YB, Su H and Qu QM: SIRT3 Protects rotenone-induced Injury in SH-SY5Y cells by promoting autophagy through the LKB1-AMPK-mTOR pathway. Aging Dis 9: 273-286, 2018.

30. Schön MP and Erpenbeck L: The interleukin-23/Interleukin-17 axis links adaptive and innate immunity in psoriasis. Front Immunol 9: 1323, 2018.
31. Langrish CL, Chen Y, Blumenschein WM, Mattson J, Basham B, Sedgwick JD, McClanahan T, Kastelein RA and Cua DJ: IL-23 drives a pathogenic $\mathrm{T}$ cell population that induces autoimmune inflammation. J Exp Me 201: 233-240, 2005.

32. Li H, Yao Q, Mariscal AG, Wu X, Hülse J, Pedersen E, Helin K, Waisman A, Vinkel C, Thomsen SF, et al: Epigenetic control of IL-23 expression in keratinocytes is important for chronic skin inflammation. Nat Commun 9: 1420, 2018.

33. Lee J, Rodero MP, Patel J, Moi D, Mazzieri R and Khosrotehrani K: Interleukin-23 regulates interleukin-17 expression in wounds, and its inhibition accelerates diabetic wound healing through the alteration of macrophage polarization. FASEB J 32: 2086-2094, 2018.

34. Kopp T, Riedl E, Bangert C, Bowman EP, Greisenegger E, Horowitz A, Kittler H, Blumenschein WM, McClanahan TK, Marbury T, et al: Clinical improvement in psoriasis with specific targeting of interleukin-23. Nature 521: 222-226, 2015.

35. Terui T, Kobayashi S, Okubo Y, Murakami M, Hirose K and Kubo H: Efficacy and safety of guselkumab, an anti-interleukin 23 monoclonal antibody, for palmoplantar pustulosis: A randomized clinical trial. JAMA Dermatol 154: 309-316, 2018.

36. Feldmeyer L, Mylonas A, Demaria O, Mennella A, Yawalkar N, Laffitte E, Hohl D, Gilliet M and Conrad C: Interleukin 23-helper $\mathrm{T}$ cell 17 axis as a treatment target for pityriasis rubra pilaris. JAMA Dermatol 153: 304-308, 2017.

37. Rai S, Arasteh M, Jefferson M, Pearson T, Wang Y, Zhang W, Bicsak B, Divekar D, Powell PP, Naumann P, et al: The ATG5-binding and coiled coil domains of ATG16L1 maintain autophagy and tissue homeostasis in mice independently of the WD domain required for LC3-associated phagocytosis. Autophagy 15: 599-612, 2019.

38. Cecconi $\mathrm{F}$ and Levine $\mathrm{B}$ : The role of autophagy in mammalian development: Cell makeover rather than cell death. Dev Cell 15: 344-357, 2008

39. Bjørkøy G, Lamark T, Brech A, Outzen H, Perander M, Overvatn A, Stenmark H and Johansen T: p62/SQSTM1 forms protein aggregates degraded by autophagy and has a protective effect on huntingtin-induced cell death. J Cell Biol 171: 603-614, 2005.

40. Pankiv S, Clausen TH, Lamark T, Brech A, Bruun JA, Outzen H, Øvervatn A, Bjørkøy G and Johansen T: p62/SQSTM1 binds directly to Atg8/LC3 to facilitate degradation of ubiquitinated protein aggregates by autophagy. J Biol Chem 282: 24131-24145, 2007.

41. Zimmermann K, Baldinger J, Mayerhofer B, Atanasov AG and Dirsch VM: Activated AMPK boosts the Nrf2/HO-1 signaling axis-A role for the unfolded protein response. Free Radic Biol Med 88: 417-426, 2015.

42. Wen J, Xu B, Sun Y, Lian M, Li Y, Lin Y, Chen D and Diao Y: Paeoniflorin protects against intestinal ischemia/reperfusion by activating LKB1/AMPK and promoting autophagy. Pharmacol Res 146: 104308, 2019.

43. Li YC, Qiao JY, Wang BY, Bai M, Shen JD and Cheng YX: Paeoniflorin ameliorates fructose-induced insulin resistance and hepatic steatosis by activating LKB1/AMPK and AKT pathways. Nutrients 10: 1024, 2018.

This work is licensed under a Creative Commons Attribution-NonCommercial-NoDerivatives 4.0 International (CC BY-NC-ND 4.0) License. 